\title{
A phase II trial of dose-reduced nab-paclitaxel for patients with previously treated, advanced or recurrent gastric cancer (OGSG 1302)
}

\author{
Shigeyuki Tamura ${ }^{1}$ [ Hirokazu Taniguchi ${ }^{2} \cdot$ Kazuhiro Nishikawa $^{3} \cdot$ Hiroshi Imamura $^{4} \cdot$ Junya Fujita $^{5} \cdot$ \\ Atsushi Takeno ${ }^{6}$. Jin Matsuyama ${ }^{7}$. Yutaka Kimura ${ }^{8}$. Junji Kawada ${ }^{1} \cdot$ Motohiro Hirao $^{3}$. Masashi Hirota ${ }^{4}$. \\ Kazumasa Fujitani ${ }^{9} \cdot$ Yukinori Kurokawa $^{10}$. Daisuke Sakai ${ }^{11}$. Hisato Kawakami ${ }^{12}$. Toshio Shimokawa ${ }^{13}$. \\ Taroh Satoh ${ }^{11}$
}

Received: 31 May 2020 / Accepted: 6 August 2020 / Published online: 14 September 2020

(c) The Author(s) 2020

\begin{abstract}
Background For unresectable or recurrent advanced gastric adenocarcinoma (AGC), tri-weekly administration of nanoparticle albumin-bound paclitaxel ( $n a b-\mathrm{PTX})$ at $260 \mathrm{mg} / \mathrm{m}^{2}$ achieved a response rate of $27.8 \%$ in a phase II trial in Japan. However, frequent neutropenia and peripheral neuropathy limit its use in clinical settings. We, thus, conducted a single-arm phase II trial to investigate the efficacy and safety of a reduced dose $\left(220 \mathrm{mg} / \mathrm{m}^{2}\right)$ of tri-weekly $n a b$-PTX.

Methods Eligible patients included those with AGC and ECOG performance status of 0-2 who had received one or more prior chemotherapy containing fluoropyrimidine regimens. A reduced dose of $n a b$-PTX $\left(220 \mathrm{mg} / \mathrm{m}^{2}\right)$ was administered triweekly. The primary endpoint was response rate (RR). Secondary endpoints were overall survival (OS), progression-free survival (PFS), disease-control rate (DCR), incidence of adverse events, relative dose intensity (RDI) and proportion of patients receiving subsequent chemotherapy.

Results Among 33 patients enrolled, 32 were treated with protocol therapy. RR was 3.1\% [95\% confidence interval (CI), $0-16.2 \%$ ], which did not reach the protocol-specified threshold $(p=0.966)$. DCR was 37.5\% (95\% CI, 21.1-56.3\%). Median OS and PFS were 6.3 (95\% CI, 4.4-14.2) and 2.2 (95\% CI, 1.8-3.1) months, respectively. RDI was 97.8\%. Twenty (62.5\%) patients received subsequent chemotherapy. Toxicity was relatively mild with the most common grade $\geq 3$ adverse events being neutropenia (38\%), anemia (13\%), fatigue (19\%), anorexia (16\%), and peripheral neuropathy (13\%).

Conclusion Tri-weekly $n a b$-PTX with a reduced dose $\left(220 \mathrm{mg} / \mathrm{m}^{2}\right)$ is not recommended for AGC in a second-line or later setting, despite demonstrating less toxicity than at $260 \mathrm{mg} / \mathrm{m}^{2}$.

Clinical trial registration

The OGSG1302 trial was registered with UMIN-CTR as UMIN000000714.
\end{abstract}

Keywords Advanced gastric cancer $\cdot$ Recurrent gastric cancer $\cdot N a b$-paclitaxel $\cdot$ Paclitaxel

\section{Introduction}

Combination of chemotherapy with platinum agents and fluoropyrimidine has been regarded as the standard of care in a first-line setting for unresectable or recurrent advanced gastric adenocarcinoma (AGC) [1], to which trastuzumab, an anti-HER2 monoclonal antibody, is added in HER2-positive

Shigeyuki Tamura

shigeyuki.tamura@hosp-yao.osaka.jp

Extended author information available on the last page of the article cases [2, 3]. Recently, oral fluoropyrimidine including S-1 and capecitabine, has generally been utilized instead of infusional 5-FU because of their convenience and tolerability [4-6]. The combination of S-1 and cisplatin is now accepted as the standard regimen for first-line chemotherapy for patients with AGC in Japan, based on the result of the SPIRITS trial [4].

For second-line chemotherapy, until weekly solvent-based paclitaxel ( $s b$-PTX) plus ramucirumab, an anti-VEGFR antibody, demonstrated superiority over $s b$-PTX alone [7], $s b$-PTX alone was widely utilized in this setting based on a phase III study in which $s b$-PTX showed comparable 
efficacy to CPT-11 with less toxicity [8]. This was also supported by promising results of several phase II studies, yielding overall response rates (RRs) that ranged from 16 to $27 \%$ and overall survival (OS) times of 5-11 months [8-12]. However, $s b$-PXT can cause hypersensitivity and anaphylactic reactions in certain patients, mostly because of polyethoxylated castor oil contained in it [13]. To use this drug safely, premedication with steroids and histamine $\mathrm{H}-2$ blockers is generally required. Moreover, $s b$-PTX contains alcohol. These factors limit the general use of $s b$-PTX in a certain subset of AGC patients.

Nanoparticle albumin-bound paclitaxel (nab-PXT) is a novel, biologically interactive, nanometer-size albuminbound paclitaxel particle initially developed to avoid the toxicities associated with polyethoxylated castor oil. It can be administered as a high dose of paclitaxel without premedication with steroids and histamine H-2 blockers. Furthermore, $n a b$-PTX can be administered in only $30 \mathrm{~min}$ and used safely for alcohol-intolerant patients [14]. In clinical trials for metastatic breast cancer (MBC) as well as non-small-cell lung cancer, $n a b$-PTX demonstrated efficacy equivalent to or exceeding that of $s b$-PTX [15-17]. In patients with AGC, a phase II trial in Japan showed the efficacy of tri-weekly nabPTX at $260 \mathrm{mg} / \mathrm{m}^{2}$ without anti-allergic premedication, with an overall RR, the primary endpoint of this study, of $27.8 \%$ [15/54; 95\% confidence interval (CI), 16.5-41.6\%] and the median progression-free survival (PFS) and OS being 2.9 (95\% CI, 2.4-3.6) and 9.2 (95\% CI, 6.9-11.4) months, respectively [18]. However, relatively high toxicity was indicated, with the most common grade $3 / 4$ toxicities being neutropenia (49.1\%), leucopenia (20.0\%), lymphopenia (10.9\%) and chemotherapy-induced peripheral neuropathy (CIPN) (23.6\%) [18]. Against this background, the optimal dosing that can minimize toxicity without sacrificing anticancer efficacy remains to be established.

A phase I trial of $n a b$-PTX in patients with advanced solid tumors determined the maximum tolerated dose to be $300 \mathrm{mg} / \mathrm{m}^{2}$ [19]. In MBC, the dose of $n a b$-PTX was initially set as $300 \mathrm{mg} / \mathrm{m}^{2}$ [20], and then deduced to $260 \mathrm{mg} / \mathrm{m}^{2}$ in the subsequent phase III Ca012 trial, where tri-weekly nab-PTX demonstrated significantly superior RR as well as a longer time to progression compared to the conventional $s b$-PTX at a dose of $175 \mathrm{mg} / \mathrm{m}^{2}$ [15]. This study also identified grade 3 or higher CIPN as a considerable adverse event of nab-PTX [15]. To reduce such toxicity, low-dose tri-weekly nab-PTX $\left(160-175 \mathrm{mg} / \mathrm{m}^{2}\right)$ was examined in several phase II studies for MBC, showing good overall RR (23-39.5\%) without CIPN of grade 3 or higher [21, 22].

These results reveal the need to develop a low-dose $n a b$ PTX regimen in AGC. To this end, we conducted a phase II trial to evaluate the efficacy and safety of low-dose triweekly $n a b$-PTX $\left(220 \mathrm{mg} / \mathrm{m}^{2}\right)$ in AGC patients in secondline or later setting.

\section{Patients and methods}

\section{Study objectives and design}

This study was conducted in accordance with the international ethical recommendations stated in the Declaration of Helsinki. The protocol was approved by the institutional ethics committees of each participating hospital and registered in the University Hospital Medical Information Network (UMIN) database (ID000000714). Written informed consent was obtained from each patient before enrollment.

This was a non-randomized, multicenter phase II study for patients with AGC for whom more than one regimen including fluorinated pyrimidine antineoplastic agents had failed. The primary endpoint was RR, and the secondary endpoints were OS, PFS, time to treatment failure (TTF), disease-control rate (DCR), safety, relative dose intensity and proportion of patients who received subsequent therapy. This trial was carried out in accordance with the Japanese Classification of Gastric Carcinoma of the 14th edition from the Japanese Gastric Cancer Association.

\section{Eligibility criteria}

The eligibility criteria of this study were as follows; (1) histologically confirmed unresectable or recurrent gastric or esophagogastric junction adenocarcinoma; (2) history of failure of one or more prior chemotherapy containing fluoropyrimidine regimens for HER2-negative cases or both fluoropyrimidine and Trastuzumab for HER2-positive cases; (3) age 20-80 years; (4) Eastern Cooperative Oncology Group (ECOG) performance status (PS) of 0-2; (5) one measurable lesion according to RECIST ver. 1.1 criteria as determined via computed tomography (CT) within 4 weeks before enrollment; (6) no previous treatment with PTX; (7) adequate organ function, including leukocyte count under $12,000 \mathrm{~mm}^{3}$, neutrophil count over $2,000 \mathrm{~mm}^{3}$, platelet count over $100,000 \mathrm{~mm}^{3}$, hemoglobin level over $9.0 \mathrm{~g} / \mathrm{dl}$, serum bilirubin level under $1.5 \mathrm{mg} / \mathrm{dl}$, aspartate aminotransferase (AST) and alanine aminotransferase (ALT) levels of under 100 or $200 \mathrm{IU} / \mathrm{L}$ of patients with liver metastasis, a serum creatinine level under $1.5 \mathrm{mg} / \mathrm{dl}$; (8) expected to survive for at least 90 days from the date of registration; and (9) cases with the provision of informed consent.

Exclusion criteria were as follows: (1) with a history of severe drug sensitivity; (2) with infection or suspected infection with a fever over $38.0^{\circ} \mathrm{C}$; (3) serious complications, such as interstitial pneumonia or lung fibrosis, uncontrolled diabetes, or renal or hepatic failure; (4) suffering more than four bouts of diarrhea; (5) a history or 
complication of heart disease, for example, congestive heart failure, myocardial infarction, ischemic heart disease requiring treatment, arrhythmia or valvular disease; (6) active double cancer; (7) peripheral neuropathy over grade 2 ; (8) difficulty enrolling due to a psychiatric or neurological disorders; (9) brain metastasis; (10) positivity for HBs antigen or HCV antibody, and (11) the a presence of any other condition that would make the treatment unsafe.

Written informed consent was obtained from each patient before enrollment and the protocol was approved by the institutional ethics committee of each participating centers.

\section{Study design}

\section{Treatment}

$n a b$-PTX was administered intravenously on an outpatient basis by a 30-min infusion at a dose of $220 \mathrm{mg} / \mathrm{m}^{2}$ on day 1 of each 21-day cycle. No premedication, such as steroid or antihistamine premedication, was administered. Treatment was continued until disease progression, unacceptable toxicity, or consent withdrawal.

Two dose-reduction levels (level $1,180 \mathrm{mg} / \mathrm{m}^{2}$ and level $\left.2,150 \mathrm{mg} / \mathrm{m}^{2}\right)$ and one dose escalation level $\left(260 \mathrm{mg} / \mathrm{m}^{2}\right)$ were implemented under the dose-reduction or escalation criteria: if the number of neutrophils was $1500 / \mathrm{mm}^{3}$ or more after the administration of $220 \mathrm{mg} / \mathrm{m}^{2} n a b$-PTX in the previous course and the dose-reduction criteria were not violated, the dose of $n a b$-PTX could be increased up to $260 \mathrm{mg} / \mathrm{m}^{2}$ in the next course.

\section{Follow-up}

Patients underwent hematological tests and assessments of clinical symptoms at least once during each course of chemotherapy. However, in the first course, hematological tests were conducted on the 1st, 8th and 15th day. The severity of adverse drug reactions was judged in accordance with the National Cancer Institute Common Terminology Criteria for Adverse Events, version 3.0. Thoracoabdominal CT scans were repeated at least every 6 weeks ( \pm 2 weeks) after treatment initiation and at the end of the treatment in this study. The objective disease status was assessed in accordance with the RECIST guidelines, version1.1. An independent review board organized by the Osaka Gastrointestinal Cancer Chemotherapy Study Group (OGSG) objectively identified treatment responses and drug-related adverse events.

\section{Statistical analysis}

RR was reported to be $27.8 \%$ (95\% CI: $16.5-41.3$ ) in a phase II study of nab-PTX $\left(260 \mathrm{mg} / \mathrm{m}^{2}, \mathrm{q} 3 \mathrm{w}\right)$ with almost the same objective as the present study [18] and $23 \%$ in a phase II study of PTX (210 mg/m²; 95\% CI: 13-36\%) [9]. The calculation of the sample size for the study was based on an expected response rate of $25 \%$ and a threshold response rate of $10 \%$, using a one-sided alpha error of 0.05 and statistical power of $80 \%$. The planned sample size was 35 patients, allowing for four patients dropping out.

The analysis focused on patients who were enrolled in this study and received at least one course of $n a b$-PTX treatment. Background data were summarized as frequency with proportion for categorical variables, and median with range for continuous variables. The response rate was evaluated using exact binomial test. Confidence intervals of response rate and disease-control rate were estimated by the Clopper-Pearson method. OS, PFS and TTF were estimated using the Kaplan-Meier method and the 95\% CIs for survival rate were calculated using Greenwood's formula. $p$ values less than 0.05 were considered statistically significant. All statistical analyses were performed with S-plus version 3.6.1 (R Foundation for Statistical Computing, Vienna Austria).

\section{Results}

Between April 2014 and December 2018, 33 patients with AGC and ECOG PS of $0-2$ who had received one or more prior chemotherapy containing fluoropyrimidine regimens were enrolled from 10 institutions in Japan. As one patient withdrew consent before the initial treatment, 32 patients received the study treatment and were evaluated for clinical response and safety. The patients' characteristics are listed in Table 1. Twenty-seven patients were male (84.4\%) and the median age was 70 years (range 48-82). Most of the patients had an ECOG PS of 0 or 1, whereas ECOG PS 2 was seen in two patients $(6 \%)$. Twenty patients involved advanced cases and 12 involved relapse. The stages at initial treatment of the relapse cases were stage II for 1 patient, stage III for 9 , and stage IV for 2 . Twenty-three patients $(72 \%)$ were enrolled as second-line treatment and nine patients $(28 \%)$ as third-line treatment.

\section{Efficacy}

The overall responses in the 32 patients are summarized in Table 2. Partial response was achieved in only one patient, yielding an overall RR of 3.1\% (95\% CI, 0-16.2\%), which did not reach the protocol-specified threshold $(p=0.966)$. Stable disease (SD) was observed in 11 patients, providing a DCR of $37.5 \%$ (95\% CI, 21.1-56.3\%). At the data cut-off (February 2019), the median follow-up was 6.3 months, and the number of treatment courses administered ranged from 1 to 27 , with a median of 3 . Only 1 out of 32 patients increased their treatment dose to $260 \mathrm{mg} / \mathrm{m}^{2}$ in the second course. The median PFS was 2.2 months (95\% CI, 
Table 1 Baseline characteristics of the patients

\begin{tabular}{|c|c|}
\hline & $n=32$ \\
\hline \multicolumn{2}{|l|}{ Gender } \\
\hline Male & $27(84 \%)$ \\
\hline Female & $5(16 \%)$ \\
\hline \multicolumn{2}{|l|}{ Age } \\
\hline Median (range) & $70(48-82)$ \\
\hline \multicolumn{2}{|c|}{ ECOG performance status } \\
\hline 0 & $18(56 \%)$ \\
\hline 1 & $12(38 \%)$ \\
\hline 2 & $2(6 \%)$ \\
\hline \multicolumn{2}{|l|}{ Body mass index } \\
\hline Median (range) & $20.0(16.0-27.0)$ \\
\hline \multicolumn{2}{|l|}{ Diagnosis } \\
\hline Advanced & $20(63 \%)$ \\
\hline Relapse & $12(38 \%)$ \\
\hline \multicolumn{2}{|l|}{ Previous gastrectomy } \\
\hline Yes & $5(16 \%)$ \\
\hline No & $27(84 \%)$ \\
\hline \multicolumn{2}{|l|}{ Metastatic site } \\
\hline None & $10(31 \%)^{\mathrm{a}}$ \\
\hline Liver & $7(22 \%)$ \\
\hline Lymph nodes & $4(13 \%)$ \\
\hline Liver + peritoneum & $3(9 \%)$ \\
\hline Peritoneum & $7(22 \%)$ \\
\hline Liver + lymph node & $1(3 \%)$ \\
\hline \multicolumn{2}{|c|}{ Number of previous chemotherapy regimens } \\
\hline 1 & $23(72 \%)$ \\
\hline 2 & $9(28 \%)$ \\
\hline \multicolumn{2}{|c|}{ Previous systemic anticancer agents } \\
\hline S-1 & $25(78 \%)$ \\
\hline CDDP & $18(56 \%)$ \\
\hline Capecitabine & $7(22 \%)$ \\
\hline CPT-11 & $6(19 \%)$ \\
\hline Oxaliplatin & $6(19 \%)$ \\
\hline Docetaxel & $2(6 \%)$ \\
\hline Other $^{b}$ & $4(13 \%)$ \\
\hline
\end{tabular}

Data are $n(\%)$

${ }^{\mathrm{a}}$ All 10 patients were relapsed cases.

${ }^{\mathrm{b}}$ Other included two patients with UFT (tegafur + uracil), one with TAS118 (S-1 + leucovorin) and one with trastuzumab.

1.8-3.1) with the 6-month PFS rate being 9.4\% (95\% CI, 3.2-27.5\%; Fig. 1). The median TTF was 2.0 months (95\% CI, 1.8-3.0, Fig. 2). The RDI was $97.8 \%$ (average dose of $\left.215 \mathrm{mg} / \mathrm{m}^{2}\right)$. The median OS was 6.4 months $(95 \% \mathrm{CI}$, 4.4-14.2) and the 1-year survival rate was $34.4 \%$ (95\% CI, 21.3\%-55.5\%: Fig. 3). Subsequent chemotherapy was received by 20 of the 32 patients $(62.5 \%$; Table 3$)$, in which the most commonly selected regimen was CPT-11based chemotherapy $(60 \%)$.
Table 2 Clinical responses

\begin{tabular}{llll}
\hline & $n$ & $\%$ & $95 \%$ CI \\
\hline Complete response (CR) & 0 & 0 & \\
Partial response (PR) & 1 & 3.1 & \\
Stable disease (SD) & 11 & 34.4 & \\
Progressive disease (PD) & 16 & 50.0 & \\
Not evaluated & 4 & 12.5 & \\
Response rate (RR) & 1 & $3.1 *$ & $0.0-16.2 \%$ \\
Disease control rate (DCR): & 12 & 37.5 & $21.1-56.3 \%$ \\
$\quad$ CR + PR + SD & & & \\
$*$ & & & \\
&
\end{tabular}

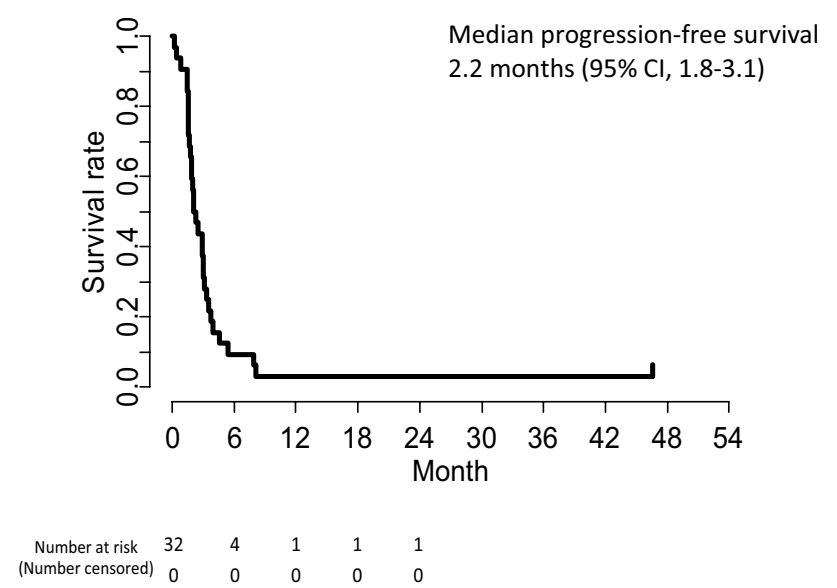

Fig. 1 Kaplan-Meier curve of progression-free survival of patients with advanced or recurrent gastric cancer receiving tri-weekly nanoparticle albumin-bound paclitaxel (nab-PTX) at a dose of $220 \mathrm{mg} / \mathrm{m}^{2}$ in a second-line or later setting

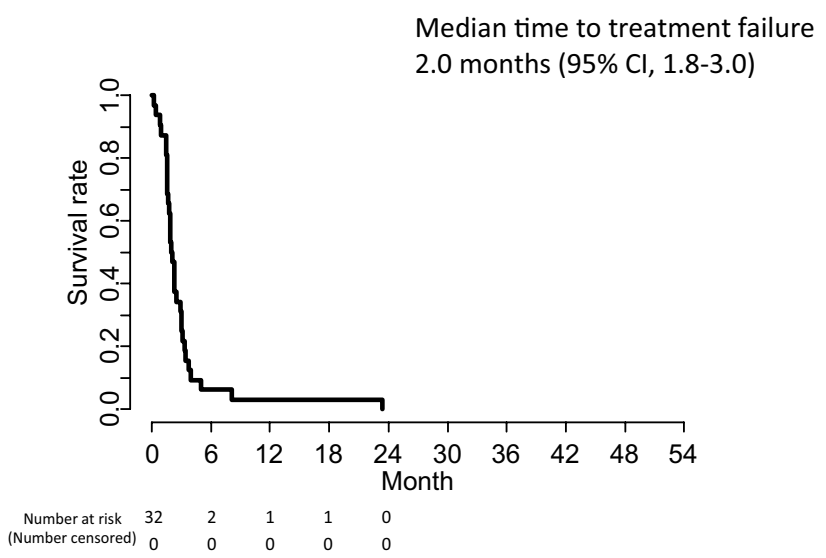

Fig. 2 Kaplan-Meier curve of time to treatment failure of patients with advanced or recurrent gastric cancer receiving nab-PTX $\left(220 \mathrm{mg} / \mathrm{m}^{2}\right)$ in a second-line or later setting 


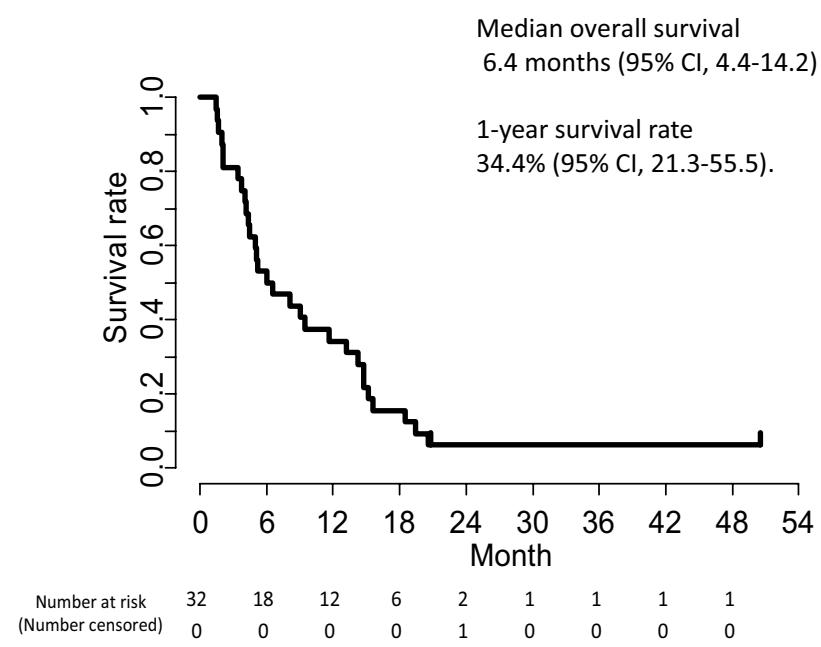

Fig. 3 Kaplan-Meier curve of overall survival of patients with advanced or recurrent gastric cancer receiving $n a b$-PTX in a secondline or later setting

Table 3 Subsequent chemotherapy after the study treatment

\begin{tabular}{lc}
\hline & $n=32$ \\
\hline Any systemic therapy & $20(63 \%)$ \\
Number of regimens & \\
1 & $12(38 \%)$ \\
2 & $6(19 \%)$ \\
3 & $2(6 \%)$ \\
Systemic anticancer agents & \\
CPT-11 & $12(38 \%)$ \\
Ramucirumab & $11(34 \%)$ \\
Paclitaxel & $4(13 \%)$ \\
Oxaliplatin & $4(13 \%)$ \\
S-1 & $3(9 \%)$ \\
Docetaxel & $2(6 \%)$ \\
Capecitabin & $2(6 \%)$ \\
Nivolumab & $2(6 \%)$ \\
\hline
\end{tabular}

Data are $n(\%)$

\section{Toxicity}

Grade 3 or 4 adverse events with an incidence rate of $>10 \%$ included neutropenia (38\%), leucopenia (13\%) and anemia $(13 \%)$ as hematological toxicities, along with fatigue (19\%), anorexia (16\%), and CIPN (13\%) as non-hematological toxicities. No patients experienced hypersensitivity or acute infusion reactions, although no premedication was administered at chemotherapy. Neither febrile neutropenia nor treatment-related deaths were observed (Table 4). The main reasons for treatment discontinuation or withdrawal were disease progression (26 cases: $81.3 \%$ ) and adverse events (4 cases: $12.5 \%)$.

\section{Discussion}

In patients with AGC, the efficacy of tri-weekly nab-PTX at $260 \mathrm{mg} / \mathrm{m}^{2}$ without anti-allergic premedication was demonstrated with an overall RR of $27.8 \%$ in a phase II trial in Japan [18]. However, relatively high toxicities, such as neutropenia and CIPN, were indicated, which was the limitation of the general use of $n a b$-PTX for patients with AGC. Therefore, we investigated the safety and efficacy of a reduced dose $\left(220 \mathrm{mg} / \mathrm{m}^{2}\right)$ of tri-weekly $n a b$-PTX for patients with AGC with PS-0, 1 plus 2 in a second-line or later setting.

At the time when the current study was ongoing, a result of the phase III ABSOLUTE study was reported [23], in which the survival benefits of tri-weekly $n a b$-PTX $\left(260 \mathrm{mg} / \mathrm{m}^{2}\right)$ or weekly $n a b$-PTX $\left(100 \mathrm{mg} / \mathrm{m}^{2}\right)$ were compared with weekly $s b$-PTX $\left(80 \mathrm{mg} / \mathrm{m}^{2}\right)$ in patients with previously treated AGC. The results showed that weekly nabPTX showed non-inferiority to weekly $s b$-PTX, whereas tri-weekly $n a b$-PTX failed to demonstrate non-inferiority to $s b$-PTX. Interestingly, the study also showed that weekly nab-PTX had considerably lower grade 3 or higher toxicity than tri-weekly $n a b$-PTX especially in terms of neutropenia (41.1 vs. $64.8 \%)$ and CIPN (2.5 vs. $20.1 \%)$. As a result of these findings, tri-weekly $n a b$-PTX $\left(260 \mathrm{mg} / \mathrm{m}^{2}\right)$ is not commonly utilized in a clinical setting in AGC.

In the current study, we observed grade 3 or higher neutropenia at a rate of $38 \%$, febrile neutropenia at $3 \%$, and grade 3 or higher CIPN at $13 \%$, whereas previous trials in AGC evaluating tri-weekly nab-PTX at $260 \mathrm{mg} / \mathrm{m}^{2}$ showed grade 3 or higher neutropenia and CIPN at rates of $49.1-64.8 \%$ and $20.1-23.6 \%$, respectively. These results suggest that tri-weekly $n a b-\mathrm{PTX}$ at $220 \mathrm{mg} / \mathrm{m}^{2}$ achieved the expected reduction of adverse events, consistent with previous trials in MBC [21, 22]

With regard to the efficacy, however, RR, the primary endpoint of this study, was only $3.1 \%$, in addition to the relatively short median PFS and OS of 2.2 and 6.3 months, respectively. In contrast, previous studies evaluating triweekly nab-PTX at $260 \mathrm{mg} / \mathrm{m}^{2}$ showed RR of $25-27.8 \%$ as well as PFS and OS of 2.9-3.8 months and 9.2-10.3 months, respectively $[18,23]$. These data indicate that the efficacy of the reduced dose of nab-PTX is not satisfactory, unlike in MBC [14, 20], despite the considerably low toxicities, so it is not recommended in clinical practice. Potential reasons for the poorer efficacy in the current study than in previous ones in AGC $[18,23]$ include the difference in the treatment line (second line or later $v s$. exclusively second line) and the relatively poor patients background in our study. 
Table 4 Adverse events related to nanoparticle albumin-bound paclitaxel in patients with advanced or recurrent gastric cancer

\begin{tabular}{|c|c|c|c|c|c|c|c|c|}
\hline & \multicolumn{4}{|c|}{ Grade } & \multicolumn{2}{|c|}{ G1-4 } & \multicolumn{2}{|c|}{ G3-4 } \\
\hline & $\overline{\mathrm{G} 1}$ & $\mathrm{G} 2$ & G3 & G4 & $n$ & $\%$ & $n$ & $\%$ \\
\hline \multicolumn{9}{|l|}{ Hematological } \\
\hline Leukopenia & 5 & 5 & 2 & 2 & 14 & 43.8 & 4 & 12.5 \\
\hline Neutropenia & 0 & 3 & 8 & 4 & 15 & 46.9 & 12 & 37.5 \\
\hline Anemia & 6 & 5 & 4 & 0 & 15 & 46.9 & 4 & 12.5 \\
\hline Thrombocytopenia & 4 & 0 & 1 & 0 & 5 & 15.6 & 1 & 3.1 \\
\hline Febrile neutropenia & 0 & 0 & 1 & 1 & 1 & 3.1 & 1 & 3.1 \\
\hline \multicolumn{9}{|l|}{ Elevation of serum } \\
\hline Creatinine & 4 & 2 & 0 & 0 & 6 & 18.8 & 0 & 0.0 \\
\hline Total bilirubin & 3 & 1 & 1 & 0 & 5 & 15.6 & 1 & 3.1 \\
\hline Aspartate aminotransferase & 9 & 2 & 2 & 0 & 13 & 40.6 & 2 & 6.3 \\
\hline Alanine aminotransferase & 5 & 3 & 1 & 0 & 9 & 28.1 & 1 & 3.1 \\
\hline Alkaline phosphatase & 0 & 1 & 0 & 0 & 1 & 3.1 & 0 & 0.0 \\
\hline Hyponatremia & 1 & 0 & 0 & 0 & 1 & 3.1 & 0 & 0.0 \\
\hline Hypoalbuminemia & 4 & 2 & 3 & 0 & 9 & 28.1 & 3 & 9.4 \\
\hline \multicolumn{9}{|l|}{ Non-hematological } \\
\hline Diarrhea & 4 & 0 & 0 & 0 & 4 & 12.5 & 0 & 0.0 \\
\hline Stomatitis & 1 & 3 & 1 & 0 & 5 & 15.6 & 1 & 3.1 \\
\hline Rash & 2 & 2 & 1 & 0 & 5 & 15.6 & 1 & 3.1 \\
\hline Nausea & 4 & 2 & 2 & 0 & 8 & 25.0 & 2 & 6.3 \\
\hline Vomiting & 4 & 0 & 0 & 0 & 4 & 12.5 & 0 & 0.0 \\
\hline Fatigue & 6 & 4 & 6 & 0 & 16 & 50.0 & 6 & 18.8 \\
\hline Anorexia & 8 & 4 & 5 & 0 & 17 & 53.1 & 5 & 15.6 \\
\hline Peripheral neuropathy & 9 & 7 & 4 & 0 & 20 & 62.5 & 4 & 12.5 \\
\hline Alopecia & 7 & 12 & 0 & 0 & 19 & 59.4 & 0 & 0.0 \\
\hline Joint pain & 3 & 0 & 0 & 0 & 3 & 9.4 & 0 & 0.0 \\
\hline Myalgia & 1 & 0 & 0 & 0 & 1 & 3.1 & 0 & 0.0 \\
\hline Dyspnea & 1 & 0 & 0 & 0 & 1 & 3.1 & 0 & 0.0 \\
\hline Limb/Trunk edema & 1 & 1 & 0 & 0 & 2 & 6.2 & 0 & 0.0 \\
\hline Fever & 2 & 1 & 0 & 0 & 3 & 9.4 & 0 & 0.0 \\
\hline Dysguesia & 1 & 0 & 0 & 0 & 1 & 3.1 & 0 & 0.0 \\
\hline
\end{tabular}

In summary, the present study failed to show a clinical benefit of a reduced dose of tri-weekly nab-PTX in AGC and indicated that such treatment is not recommended, despite being potentially less toxic than the original dosing. Tri-weekly nab-PTX has little currency in the treatment of AGC as of the ABSOLUTE study [23], whereas the weekly administration of $n a b$-PTX is now widely accepted. Following the success of the RAINBOW trial [7], weekly $n a b$-PTX in combination with ramucirumab was examined in a phase II trial [24], showing promising activity with an RR of $54.8 \%$ and manageable toxicities. Given that the clinical benefit of weekly $n a b$-PTX was pronounced in patients with peritoneal metastasis [23], a randomized phase II study comparing $s b$-PTX plus ramucirumab with nab-PTX plus ramucirumab is ongoing in AGC patients with peritoneal metastasis in a second-line setting (WJOG_P-SELECT study, jRCTs031180022), and the results of which are awaited. 
Table 5 Comparison of background factors and results

\begin{tabular}{|c|c|c|c|c|c|c|}
\hline & \multicolumn{2}{|c|}{$\begin{array}{l}\text { Phase II study }{ }^{18)}(260 \mathrm{mg} / \\
\left.\mathrm{m}^{2}\right)\end{array}$} & \multicolumn{2}{|c|}{$\begin{array}{l}\text { Absolute study }{ }^{23)}(260 \mathrm{mg} / \\
\left.\mathrm{m}^{2}\right)\end{array}$} & \multicolumn{2}{|c|}{$\begin{array}{l}\text { Current study }(220 \mathrm{mg} / \\
\left.\mathrm{m}^{2}\right)\end{array}$} \\
\hline & No. of patients & $\%$ & No. of patients & $\%$ & No. of patients & $\%$ \\
\hline No. of patients & 56 & & 243 & & 32 & \\
\hline \multicolumn{7}{|l|}{ Age } \\
\hline Median (years) & 63.5 & & 66 & & 70 & \\
\hline \multicolumn{7}{|c|}{ ECOG performance status } \\
\hline 0 & 33 & 58.9 & 167 & 69 & 18 & 56 \\
\hline 1 & 23 & 41.1 & 72 & 30 & 12 & 38 \\
\hline 2 & 0 & 0 & 4 & 2 & 2 & 6 \\
\hline \multicolumn{7}{|c|}{ No. of pretreatment regimens } \\
\hline 1 & 56 & 100 & 243 & 100 & 23 & 72 \\
\hline 2 & 0 & 0 & 0 & 0 & 9 & 28 \\
\hline \multicolumn{7}{|c|}{ Subsequent treatment } \\
\hline Any & 44 & 81.5 & $165(231)$ & 71.4 & 20 & 62.5 \\
\hline CPT-11 & 29 & 53.7 & 124 & 51 & 12 & 37.5 \\
\hline $\mathrm{ORR}^{\mathrm{a}}(\mathrm{CR}+\mathrm{PR})$ & 15 & 27.8 & $38(150)$ & 25 & 1 & 3.1 \\
\hline $\mathrm{PFS}^{\mathrm{b}}$ (months) & 2.9 & & 3.8 & & 2.2 & \\
\hline $\mathrm{PDI}^{\mathrm{c}}$ & $243 \mathrm{mg} / \mathrm{m}^{2}$ & 93.4 & $229 \mathrm{mg} / \mathrm{m}^{2}$ & 88.06 & $215 \mathrm{mg} / \mathrm{m}^{2}$ & 97.8 \\
\hline \multicolumn{7}{|c|}{ Median duration of treatment (M: months) } \\
\hline & 2.6 & & 2.4 & & 2.0 & \\
\hline OS $^{\mathrm{d}}$ (months) & 9.2 & & 10.3 & & 6.4 & \\
\hline
\end{tabular}

(): The numbers in parentheses indicate the number of cases evaluated

${ }^{a}$ Overall response rate

${ }^{\mathrm{b}}$ Progression-free survival (median)

${ }^{\mathrm{c}}$ Relative dose intensity

${ }^{\mathrm{d} O \text { Overall survival (median) }}$
Acknowledgement We thank all of the patients, investigators, and medical staff who participated in this study, as well as staff at the OGSG data center for their contribution.

Funding This study was funded by Taiho Pharmaceutical Co., Ltd. under the study contract.

\section{Compliance with ethical standards}

Conflict of interest Kazuhiro Nishikawa has received honoraria from Bristol-Myers Squibb, Chugai, EA Pharma, Eli Lilly, Ono, Taiho and Yakult. Hiroshi Imamura has received honoraria from Taiho. Kazumasa Fujitani has received honoraria from Bristol-Myers Squibb, Eli Lilly, Ono, Taiho and Yakult. Yukinori Kurokawa has received honoraria from Taiho, Ono, Eli Lilly, Yakult, Bristol-Myers Squibb, Kaken, Chugai and Takeda, and research funding from Taiho, Ono and MSD. Daisuke Sakai has received honoraria from Chugai and Diichi-Sankyo, and research funding from Chugai, Yakult, Ono, Eli Lilly and DiichiSankyo. Hisato Kawakami has received honoraria from Bristol-Myers Squibb. Astra-Zeneca, Bayer Yakuhin, Eli Lilly, MSD, Ono, Chugai, Daiichi Sankyo, Takeda and Taiho, and research funding from Chugai, Taiho and Eisai. Taroh Satoh has received honoraria from BristolMyers Squibb, Eli Lilly, Ono, Chugai and Taiho, and research funding from MSD, Gilead, Astellas and Astra-Zeneca, and have received scholarship donations from Taiho, and endowed chairs from Ono, Chugai and Yakult. All remaining authors declare no conflict of interest.
Open Access This article is licensed under a Creative Commons Attribution 4.0 International License, which permits use, sharing, adaptation, distribution and reproduction in any medium or format, as long as you give appropriate credit to the original author(s) and the source, provide a link to the Creative Commons licence, and indicate if changes were made. The images or other third party material in this article are included in the article's Creative Commons licence, unless indicated otherwise in a credit line to the material. If material is not included in the article's Creative Commons licence and your intended use is not permitted by statutory regulation or exceeds the permitted use, you will need to obtain permission directly from the copyright holder. To view a copy of this licence, visit http://creativecommons.org/licenses/by/4.0/.

\section{References}

1. Ohtsu A, Shimada Y, Shirao K et al (2003) Randomized phase III trial of fluorouracil alone versus fluorouracil plus cisplatin versus uracil and tegafur plus mitomycin in patients with unresectable, advanced gastric cancer: The Japan Clinical Oncology Group Study (JCOG9205). J Clin Oncol 21(1):54-59

2. Bang YJ, Van Cutsem E, Feyereislova A et al (2010) Trastuzumab in combination with chemotherapy versus chemotherapy alone for treatment of HER2-positive advanced gastric or gastrooesophageal junction cancer (ToGA): a phase 3, open-label, randomised controlled trial. Lancet 376(9742):687-697. https://doi. org/10.1016/S0140-6736(10)61121-X 
3. Sawaki A, Ohashi Y, Omuro Y et al (2012) Efficacy of trastuzumab in Japanese patients with HER2-positive advanced gastric or gastroesophageal junction cancer: a subgroup analysis of the Trastuzumab for Gastric Cancer (ToGA) study. Gastric Cancer 15(3):313-322. https://doi.org/10.1007/s10120-011-0118-1

4. Koizumi W, Narahara H, Hara T et al (2008) S-1 plus cisplatin versus $\mathrm{S}-1$ alone for first-line treatment of advanced gastric cancer (SPIRITS trial): a phase III trial. Lancet Oncol 9(3):215-221. https://doi.org/10.1016/S1470-2045(08)70035-4

5. Cunningham D, Starling N, Rao S et al (2008) Capecitabine and oxaliplatin for advanced esophagogastric cancer. N Engl J Med 358(1):36-46. https://doi.org/10.1056/NEJMoa073149

6. Kang YK, Kang WK, Shin DB et al (2009) Capecitabine/cisplatin versus 5-fluorouracil/cisplatin as first-line therapy in patients with advanced gastric cancer: a randomised phase III noninferiority trial. Ann Oncol 20(4):666-673. https://doi.org/10.1093/annonc/ $\operatorname{mdn} 717$

7. Wilke H, Muro K, Van Cutsem E et al (2014) Ramucirumab plus paclitaxel versus placebo plus paclitaxel in patients with previously treated advanced gastric or gastro-oesophageal junction adenocarcinoma (RAINBOW): a double-blind, randomised phase 3 trial. Lancet Oncol 15(11):1224-1235. https://doi.org/10.1016/ S1470-2045(14)70420-6

8. Hironaka S, Ueda S, Yasui H et al (2013) Randomized, openlabel, phase III study comparing irinotecan with paclitaxel in patients with advanced gastric cancer without severe peritoneal metastasis after failure of prior combination chemotherapy using fluoropyrimidine plus platinum: WJOG 4007 Trial. J Clin Oncol 31(35):4438-4444. https://doi.org/10.1200/jco.2012.48.5805

9. Yamada Y, Shirao K, Ohtsu A et al (2001) Phase II trial of paclitaxel by three-hour infusion for advanced gastric cancer with short premedication for prophylaxis against paclitaxel-associated hypersensitivity reactions. Ann Oncol 12(8):1133-1137. https:// doi.org/10.1023/a:1011680507956

10. Yamaguchi K, Tada M, Horikoshi N et al (2002) Phase II study of paclitaxel with 3-h infusion in patients with advanced gastric cancer. Gastric Cancer 5(2):90-95. https://doi.org/10.1007/s1012 00200015

11. Hironaka S, Zenda S, Boku N et al (2006) Weekly paclitaxel as second-line chemotherapy for advanced or recurrent gastric cancer. Gastric Cancer 9(1):14-18. https://doi.org/10.1007/s1012 0-005-0351-6

12. Kodera Y, Ito S, Mochizuki Y et al (2007) A phase II study of weekly paclitaxel as second-line chemotherapy for advanced gastric cancer (CCOG0302 study). Anticancer Res 27(4c):2667-2671

13. Gelderblom H, Verweij J, Nooter K et al (2001) Cremophor EL: the drawbacks and advantages of vehicle selection for drug formulation. Eur J Cancer 37(13):1590-1598. https://doi.org/10.1016/ s0959-8049(01)00171-x

14. Henderson IC, Bhatia V (2007) Nab-paclitaxel for breast cancer: a new formulation with an improved safety profile and greater efficacy. Expert Rev Anticancer Ther 7(7):919-943. https://doi. org/10.1586/14737140.7.7.919
15. Gradishar WJ, Tjulandin S, Davidson N et al (2005) Phase III trial of nanoparticle albumin-bound paclitaxel compared with polyethylated castor oil-based paclitaxel in women with breast cancer. J Clin Oncol 23(31):7794-7803. https://doi.org/10.1200/ jco.2005.04.937

16. Rizvi NA, Riely GJ, Azzoli CG et al (2008) Phase I/II trial of weekly intravenous $130-\mathrm{nm}$ albumin-bound paclitaxel as initial chemotherapy in patients with stage IV non-small-cell lung cancer. J Clin Oncol 26(4):639-643. https://doi.org/10.1200/ jco.2007.10.8605

17. Socinski MA, Bondarenko I, Karaseva NA et al (2012) Weekly nab-paclitaxel in combination with carboplatin versus solventbased paclitaxel plus carboplatin as first-line therapy in patients with advanced non-small-cell lung cancer: final results of a phase III trial. J Clin Oncol 30(17):2055-2062. https://doi.org/10.1200/ jco.2011.39.5848

18. Sasaki Y, Nishina T, Yasui H et al (2014) Phase II trial of nanoparticle albumin-bound paclitaxel as second-line chemotherapy for unresectable or recurrent gastric cancer. Cancer Sci 105(7):812817. https://doi.org/10.1111/cas.12419

19. Ibrahim NK, Desai N, Legha S et al (2002) Phase I and pharmacokinetic study of ABI-007, a cremophor-free, protein-stabilized, nanoparticle formulation of paclitaxel. Clin Cancer Res 8(5):1038-1044

20. Ibrahim NK, Samuels B, Page R et al (2005) Multicenter phase II trial of ABI-007, an albumin-bound paclitaxel, in women with metastatic breast cancer. J Clin Oncol 23(25):6019-6026. https:// doi.org/10.1200/jco.2005.11.013

21. Takashima T, Kawajiri H, Nishimori T et al (2018) Safety and efficacy of low-dose nanoparticle albumin-bound paclitaxel for HER2-negative metastatic breast cancer. Anticancer Res 38(1):379-383. https://doi.org/10.21873/anticanres. 12233

22. Yamamoto S, Maeda N, Nagashima Y et al (2017) A phase II, multicenter, single-arm study of tri-weekly low-dose nanoparticle albumin-bound paclitaxel chemotherapy for patients with metastatic or recurrent breast cancer. Breast Cancer 24(6):783-789. https://doi.org/10.1007/s12282-017-0779-7

23. Shitara K, Takashima A, Fujitani K et al (2017) Nab-paclitaxel versus solvent-based paclitaxel in patients with previously treated advanced gastric cancer (ABSOLUTE): an open-label, randomised, non-inferiority, phase 3 trial. Lancet Gastroenterol Hepatol 2(4):277-287. https://doi.org/10.1016/s2468 $-1253(16) 30219-9$

24. Bando H, Shimodaira H, Fujitani K et al (2018) A phase II study of nab-paclitaxel in combination with ramucirumab in patients with previously treated advanced gastric cancer. Eur J Cancer 91:86-91. https://doi.org/10.1016/j.ejca.2017.11.032

Publisher's Note Springer Nature remains neutral with regard to jurisdictional claims in published maps and institutional affiliations. 


\section{Affiliations}

\section{Shigeyuki Tamura ${ }^{1}$ (D) Hirokazu Taniguchi ${ }^{2} \cdot$ Kazuhiro Nishikawa $^{3} \cdot$ Hiroshi Imamura $^{4} \cdot$ Junya Fujita $^{5}$. Atsushi Takeno ${ }^{6}$. Jin Matsuyama ${ }^{7}$. Yutaka Kimura ${ }^{8}$. Junji Kawada ${ }^{1} \cdot$ Motohiro Hirao $^{3}$. Masashi Hirota ${ }^{4}$. Kazumasa Fujitani ${ }^{9} \cdot$ Yukinori Kurokawa ${ }^{10}$. Daisuke Sakai ${ }^{11}$. Hisato Kawakami ${ }^{12}$. Toshio Shimokawa ${ }^{13}$. Taroh Satoh ${ }^{11}$}

1 Department of Surgery, Yao Municipal Hospital, 1-3-1, Ryuge, Yao, Osaka 581-0069, Japan

2 Department of Gastroenterological Surgery, Osaka Saiseikai Senri Hospital, Suita, Osaka, Japan

3 Department of Surgery, National Hospital Organization Osaka National Hospital, Osaka, Japan

4 Department of Surgery, Toyonaka Municipal Hospital, Toyonaka, Osaka, Japan

5 Department of Surgery, Sakai City Medical Center, Sakai, Osaka, Japan

6 Department of Surgery, Kansai Rosai Hospital, Amagasaki, Hyogo, Japan

7 Department of Gastroenterological Surgery, Higashiosaka City Medical Center, Higashiosaka, Osaka, Japan
8 Department of Surgery, Kindai University Hospital, Sayama, Osaka, Japan

9 Department of Surgery, Osaka General Medical Center, Osaka, Japan

10 Department of Gastroenterological Surgery, Osaka University Graduate School of Medicine, Suita, Osaka, Japan

11 Department of Frontier Science for Cancer and Chemotherapy, Osaka University Graduate School of Medicine, Suita, Osaka, Japan

12 Department of Medical Oncology, Faculty of Medicine, Kindai University, Sayama, Osaka, Japan

13 Clinical Study Support Center, Wakayama Medical University, Wakayama, Japan 